\title{
Relationships between B-mode Ultrasound Imaging Signals and Suspended Sediment Concentrations
}

\author{
Xianjian Zou ${ }^{1,2}$, Huan Song ${ }^{2}$, Chuanying Wang ${ }^{1}$, Zhimin $\mathrm{Ma}^{2}$ \\ 1. State Key Laboratory of Geomechanics and Geotechnical Engineering, Institute of Rock and Soil Mechanics, \\ Chinese Academy of Sciences, 430071, Wuhan, People's Republic of China \\ 2. School of Electronic Information, Wuhan University, 430072, Wuhan, People's Republic of China \\ Corresponding author: Xianjian Zou, E-mail: zouxianjian@whu.edu.cn
}

\begin{abstract}
Ultrasound imaging technique is applied to river model test in recent years, but the imaging features and inner relationships of sediments are still waiting for further study. This paper proposes some new relationships between B-mode ultrasound imaging signals and suspended sediment concentrations (SSC) after kinds of model experiments are conducted in a glass flume. And the influences of particle size, sediment material, ultrasonic frequency and flow velocity on the relationships are discussed in practical applications. Results show that the imaging area concentration (IAC), gray area concentration (GAC) and gray energy density (GED) of sediment imaging signals increase with the addition of sediments until images are filled with imaging signals. The GAC is the best one because of the widest variation range and better sensitivity when compared with the IAC. The GED is the most sensitive to the change of SSC, but it is easiest to reach saturation. The relationship between the GAC and actual SSC can be used to estimate unknown SSC and its vertical distribution when the concentration is below $4.0 \%$ with advantages of direct observation and real time.
\end{abstract}

Key words: B-mode ultrasound device; imaging signal analysis ; river model test; relationship research; suspended sediment concentration.

\section{Introduction}

With the development of ultrasound imaging technique, some ultrasound imaging devices have been applied to the measurements of experimental parameters in river model test, such as ultrasonic velocimetry of flows [1-3] and imaging measurement of model topography [4]. All of these refer to suspended sediment particles in sediment-laden flow. Understanding the relationship between ultrasound imaging signals and suspended sediment concentration (SSC) is necessary in addressing many problems, such as the analysis of SSC and its vertical distribution [5], which is vital for the research of sediment movement and river model test.

Over the past three decades, the application of acoustics to the measurement of small-scale sediment transport processes has been gaining increasing acceptance. Acoustic instrumentations, including a triple frequency acoustic backscatter system (ABS) [6, 7], an acoustic Doppler velocity profiler (ADVP) [8], a sand ripple imager (SRI) and a sand ripple profiler (SRP), are used to estimate near-bed sediment transport processes and hydrodynamics [9]. Acoustics has the potential ability to measure non-intrusively, with high temporal and spatial resolution, profiles of suspended sediment size and concentration, profiles of flow and bed form morphology [10]. The possibility of focusing images for small sands in sediment-laden flow has come true with the 
development of modern ultrasound imaging technology [11, 12]. Using B-mode ultrasound device [13-15] to show moving sediments in the water has the advantages of direct observation, high sensitivity and real time [16-18]. Consequently, experimental research on relationships between B-mode ultrasound imaging signals and SSCs is necessary. Addition, this requires more study for the application of estimating unknown SSC and its vertical distribution in the low silt-carrying flow.

In this paper, relationships between ultrasound imaging signals and SSCs are researched after kinds of experiments by using B-mode ultrasound device, and their applications in estimating SSC and its vertical distribution are discussed. This paper is structured as follow: Section 1 describes ultrasound imaging principle and experimental system; Section 2 illustrates different sediments experiments and analyzes the relationships between B-mode ultrasound imaging signals and actual SSCs; Section 3 proposes the application of these relationships and discusses some existed influencing factors; Finally, Section 4 concludes the whole paper.

\section{Principle and System}

\subsection{B-mode Ultrasound Imaging Principle}

As we all known, B-mode ultrasound diagnostic apparatus is widely used in medicine, and our hearts and kidneys and even blood vessels are clearly shown by using it [19]. Ultrasound imaging is based on the transmission towards human body of longitudinal acoustic waves with frequency higher than $20 \mathrm{kHz}$ (generally around 1 to $20 \mathrm{MHz}$ ) and the reception of produced echoes; the signal is created and then detected by piezo-electric effect. The ultrasound imaging principle of B-mode ultrasound device for sediment-laden flow in river model test is the same. The detector of B-mode ultrasound device generates ultrasonic waves to water, and ultrasonic waves are reflected from sediment or topographic bed. Then the detector receives their echoes. The time of passage of the ultrasonic waves is measured electronically and allows for the determination of the distance from sediments to detector. And then a profile image is formed after a series of signal processing steps within B-mode ultrasound device. So, the ultrasonic images of sands or sediments in the water are formed by this way. The B-mode ultrasound device is shown in Figs. 1.

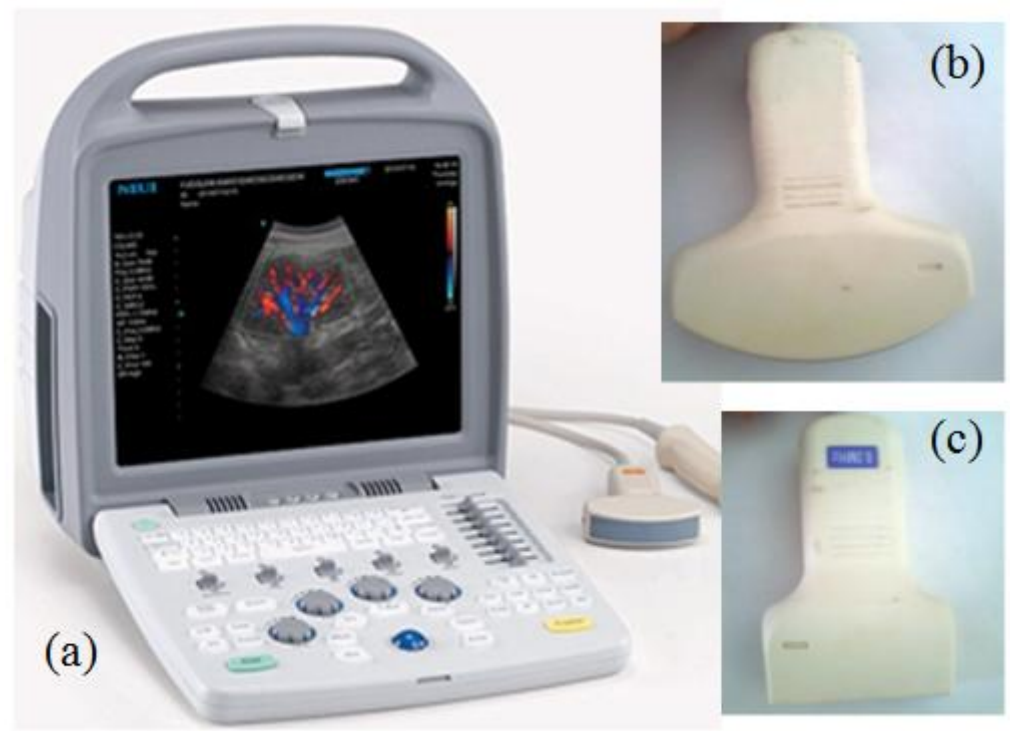

Fig. 1 B-mode ultrasound device. (a) is the device of Apogee 1100 Ultrasound Diagnostic Apparatus; (b) is the convex array detector of the device; (b) is the linear array detector of the device. 
High frequency ultrasonic waves transmitted from B-mode ultrasound device are sensitive to sediments or tiny particles underwater because of strong penetrability [20, 21]. Suspended sediments can be monitored very well, especially with the help of modern B-mode ultrasound imaging technology. B-mode ultrasound device can get images of moving particles in sediment-laden flow, and follow the change of suspended sediments, just as shown in Fig.2, which is the profile maps of sediment-laden flow including river bed.

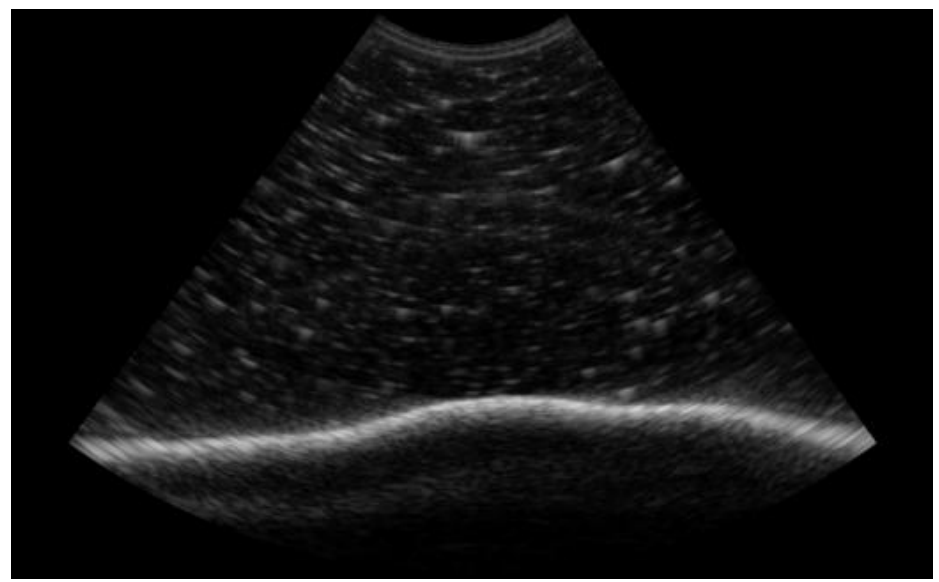

Fig. 2 B-mode ultrasound imaging of underwater sediments and topographic bed

Figure 2 is the imaging of plastic sediments with particle size $0.32 \mathrm{~mm}$ and also the imaging of topographic bed consisted of them. From Fig. 2, it is can be seen that the imaging of underwater suspended sediments and topographic bed on the bottom is clear. The imaging spots of suspended sediments or moving particles are observed very well with good self-adaption and high resolution.

\subsection{Sediments Experimental System}

In order to research the ultrasound imaging signals of suspended sediments underwater, a sediments experimental system is built up by using B-mode ultrasound device. The experimental system is consisted by an Apogee 1100 Ultrasound Diagnostic Apparatus (i.e. B-mode ultrasound device), a system computer, a mixer, a big glass flume and the software of Analysis SSCs (suspended sediment concentrations), as shown in Fig. 3.

The Apogee 1100 Ultrasound Diagnostic Apparatus is used to observe suspended sediments and to obtain the imaging signals of sediments in sediment-laden flow. In order to keep suspended sediment distribute equally as much as possible, the mixer should be run smoothly and suitable for actual needs. The detector of B-mode ultrasound device touches the water flow and transmits ultrasonic waves (e.g. choosing a middle frequency $5 \mathrm{MHz}$ ), and then acquires imaging signals of suspended sediment. The imaging signals are sent to the software for the analysis of SSCs. The software is written according to B-mode ultrasound images. It is used to receive the imaging signals, and show statistical results.

The Apogee 1100 Ultrasound Diagnostic Apparatus is an advanced B-mode ultrasound device shown in Fig. 1 (a). It has a convex array detector shown in Fig. 1 (b) and a linear array detector shown in Fig. 1 (c). The linear array detector can transmit $5 \mathrm{MHz}, 10 \mathrm{MHz}$ and $12 \mathrm{MHz}$ ultrasonic wave. The convex array detector can transmit $2.0 \mathrm{MHz}, 2.5 \mathrm{MHz}, 3.3 \mathrm{MHz}, 4.2 \mathrm{MHz}$ and 5.0 $\mathrm{MHz}$ ultrasonic wave. When the frequency of ultrasonic wave is higher, the directionality 
and resolution of ultrasonic image is higher, which is better for imaging but the attenuation of ultrasonic wave is also stronger. So, we a middle frequency $5.0 \mathrm{MHz}$ as the ultrasonic frequency. Another, because the ultrasonic beam of convex array detector is sector scanning and transmitted with wide scanning angle while linear array detector doesn't have, convex array detector has a wider vision field and a larger scanned area than linear array detector. Therefore, the convex array detector can measure more imaging area of sediment-laden flow.

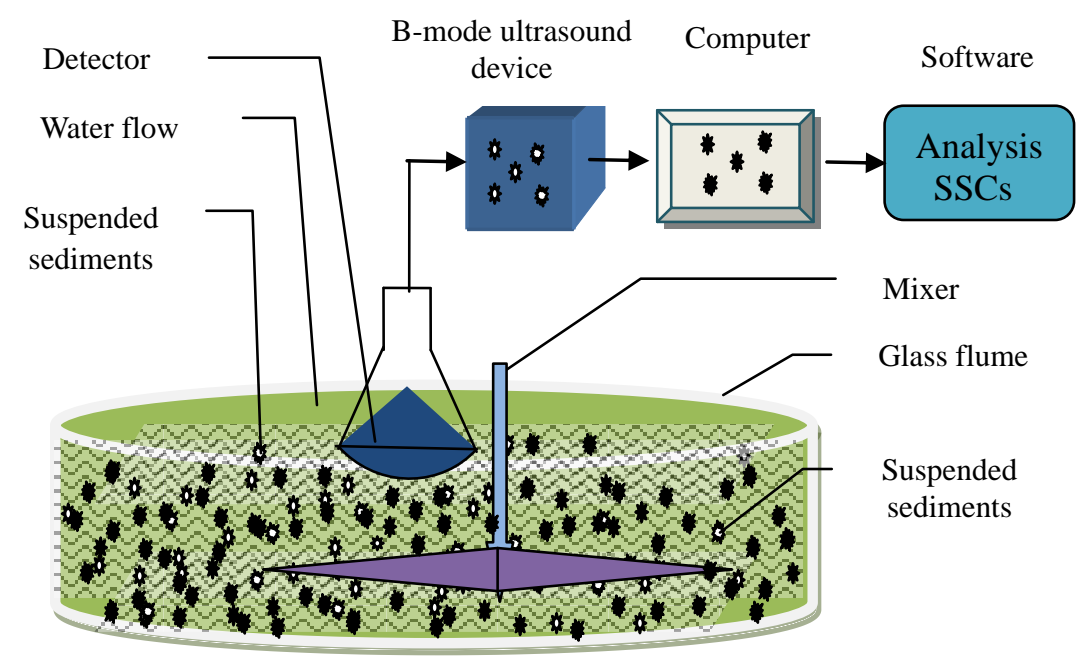

Fig. 3 Experimental system

In this paper, we choose the $5 \mathrm{MHz}$ of ultrasonic frequency and most of experiments are done by using the convex array detector. Because the Apogee 1100 Ultrasound Diagnostic Apparatus is used for medical diagnosis, its measuring depth is less than $40 \mathrm{~cm}$ and its measuring width is less than $38 \mathrm{~cm}$. Its acquisition frame frequency is from $8 \mathrm{~Hz}$ to $63 \mathrm{~Hz}$. Its measuring angle is from 10 degree to 70 degree. During the river model test, we take the same setting parameters as much as possible even under different situations. Actually, there is little difference about particle imaging signals when taking the same detector and keeping the same settings all the time, such as TGC. However, it's worth noting that a number of TGC settings from top to down are all the same as before during the whole experiments. When using B-mode ultrasound device to obtain ultrasound images of suspended sediment under the water, the right configuration of device is important for the later analysis and real-time measurement of SSC, so we keep them all right when experiments are conducted in the glass flume.

\section{Experiments and Analysis}

\subsection{Suspended Sediment Experiments}

In order to deeply understand the relationships between B-mode ultrasound imaging signals and actual SSCs, kinds of experiments are conducted in the laboratory by using the experimental system shown in Fig. 3. We take plastic sediments (PS) and natural sediments (NS) as examples. The PS is usually used in river model test. The NS is from the Yangtze River. The average particle size of PS is about $0.32 \mathrm{~mm}$. The average particle size of NS is about $0.21 \mathrm{~mm}$. The frequency of B-mode ultrasound device sets $5 \mathrm{MHz}$. And others keep the same as the first time when the sediments images are clear and able to be well analyzed. The glass flume is $10 \mathrm{~L}$. After adding PS or NS $5 \mathrm{ml}$ to it each time, it becomes volume concentration from $0.0 \%$ (tap water) to $5.0 \%$ 
when it is full of sediments in the image. At the same time, keeping the mixer running smoothly is necessary for a well-distributed sediment-laden flow. There are four typical images of suspended sediments (plastic sediments, particle size $0.32 \mathrm{~mm}$ ) experiments from B-mode ultrasound device (Apogee 1100 Ultrasound Diagnostic Apparatus) shown in Figs. 4 (profile maps of water flow without topographic bed). Figure 4 (a) is the volume concentration $0.2 \%$ of SSC. Figure 4 (b) is the volume concentration $1.0 \%$ of SSC. Figure 4 (c) is the volume concentration $3.0 \%$ of SSC. Figure $4(\mathrm{~d})$ is the volume concentration $5.0 \%$ of SSC.

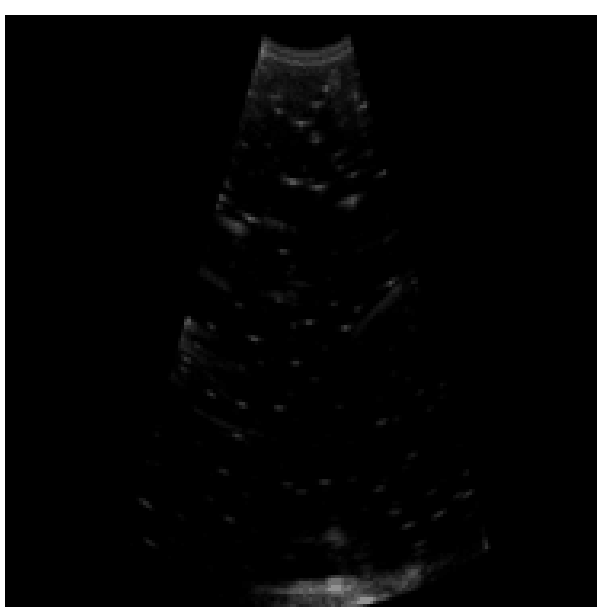

(a)

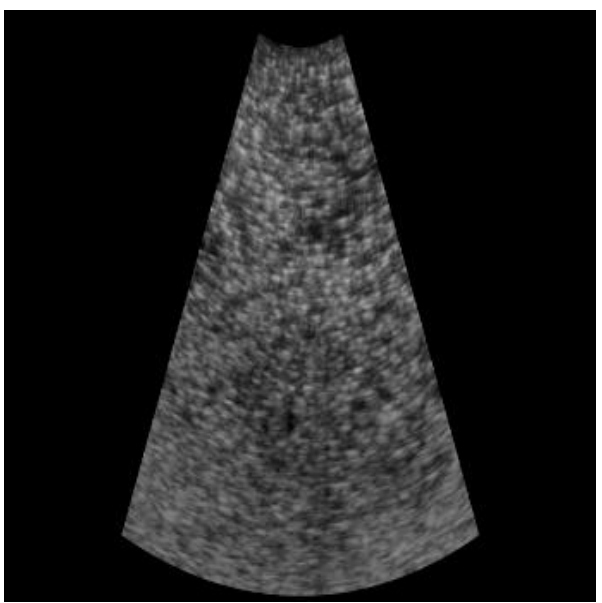

(c)

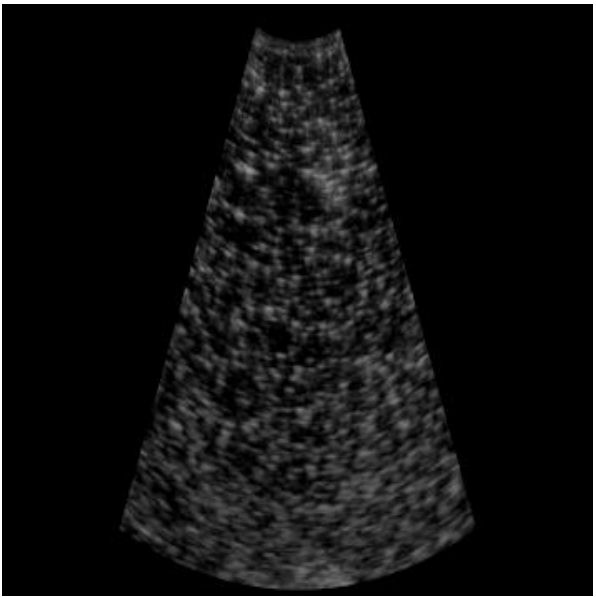

(b)

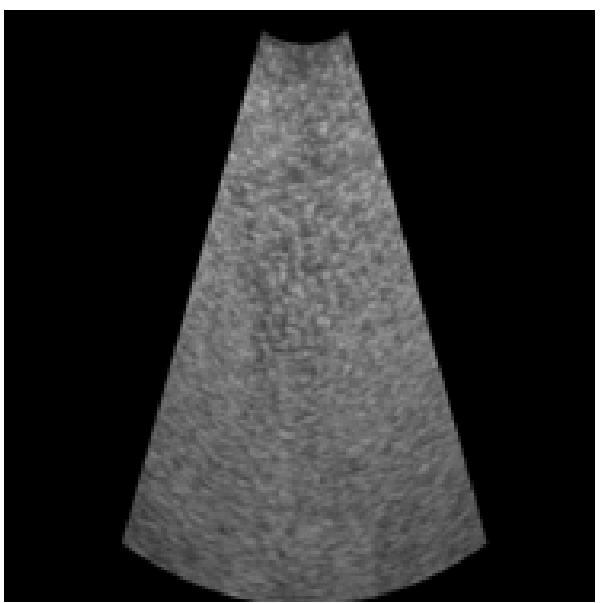

(d)

Fig. 4 Typical images of different suspended sediment concentrations (SSCs), (a) volume concentration 0.2 $\%$, (b) volume concentration $1.0 \%$, (c) volume concentration $3.0 \%$, (d) volume concentration $5.0 \%$

From Figs. 4, the actual volume concentration of suspended sediment concentrations (SSCs) in the glass flume is added from $0.2 \%$ to $5.0 \%$, and the sediment imaging spots are added as well until images are filled with sediment imaging spots. The change of sediment imaging spots can reflect the change of actual volume concentration of SSC during the experiments. So, we took some methods of statistical analysis to get their relationships. Sediment imaging signals such as imaging spots can clearly reflect a certain relationship followed with the increase of SSC in the glass flume during the experiments. The sediment imaging signals we analyzed are imaging area concentration (IAC), gray area concentration (GAC) and gray energy density (GED), which are described below. Related experimental data are shown in table 1.

The values of IAC, GAC, GED and SSC per time in table 1 are both statistical average values of 50 images every time and they are compared with PS and NS. The IAC, GAC and GED are all statistical values from two independent sediment experiments (PS and NS). 
Table 1 Relationships between imaging area concentration (IAC), gray area concentration (GAC), gray energy density (GED) and actual suspended sediments concentration (SSC) during the experiments of plastic sediments (PS) and natural sediments (NS)

\begin{tabular}{ccccccc}
\hline \multirow{2}{*}{ SSC /\%o } & \multicolumn{3}{c}{ PS /\% } & & \multicolumn{3}{c}{ NS /\% } \\
& IAC & GAC & GED & IAC & GAC & GED \\
\hline 0.0 & 0.02 & 0.01 & 0.19 & 0.05 & 0.03 & 0.47 \\
0.5 & 28.9 & 11.5 & 43.8 & 10.1 & 3.78 & 16.3 \\
1.0 & 55.1 & 22.7 & 70.7 & 30.2 & 11.4 & 40.1 \\
1.5 & 74.2 & 31.6 & 85.5 & 49.8 & 18.9 & 59.4 \\
2.0 & 86.8 & 37.4 & 93.1 & 68.1 & 25.7 & 76.1 \\
2.5 & 91.5 & 40.4 & 95.3 & 82.2 & 32.2 & 87.7 \\
3.0 & 94.1 & 42.6 & 96.6 & 88.8 & 36.3 & 92.8 \\
3.5 & 95.2 & 43.5 & 97.5 & 91.3 & 38.7 & 94.7 \\
4.0 & 96.1 & 44.2 & 98.1 & 92.3 & 39.9 & 95.7 \\
4.5 & 96.9 & 44.9 & 98.2 & 94.6 & 40.8 & 96.9 \\
5.0 & 97.7 & 45.3 & 98.8 & 95.4 & 41.6 & 97.6 \\
\hline
\end{tabular}

\subsection{Imaging Spots Features Analysis}

In this part, a detailed description for the imaging signals of suspended sediments is given after a large number of experiments performed in the glass flume of laboratory, i.e., river model test. Facing with the imaging signals, i.e. ultrasonic images obtained from B-mode ultrasound device, firstly, a whole preprocessing to the images is necessary before quantitative analysis. The original image $f(i, j)$ is processed by threshold segmentation, and then the objective image $g(i, j)$ is obtained by using following formula:

$$
g(i, j)=\left\{\begin{array}{cc}
f(i, j), & f(i, j) \geq T \\
0, & f(i, j)<T
\end{array}\right.
$$

In formula (1), the threshold value $T$ is $f_{90}$, which means a value that 90 percent of pixels' values in the imaging region are less than or equal to this value $T$.

Secondly, we define imaging area concentration (IAC), gray area concentration (GAC), gray energy density (GED) as follow:

Definition 1 The rate between the sediment imaging area and the total area of per image is called as imaging area concentration (IAC).

The IAC is a percentage in per imaging area, and it is calculated at the basis of objective image using following formula:

$$
C_{\mathrm{i}}=\frac{S_{\text {sd }}}{S_{\text {all }}}=\frac{N_{\text {sd }} S_{0}}{N_{\text {all }} S_{0}}=\frac{N_{\text {sd }}}{N_{\text {all }}} \times 100 \%
$$

Where $C_{\mathrm{i}}$ is the IAC, $S_{\text {sd }}$ is the sediment imaging area while $S_{\text {all }}$ is the total imaging area. $N_{\text {sd }}$ is the number of pixels in the sediment imaging area while $N_{\text {all }}$ is the number of pixels in the total imaging area. $\mathrm{S}_{0}$ is the area of per pixel, which is a constant value.

Definition 2 The rate between the sediment imaging area including the weighted gray information and the total area of per image is called as gray area concentration (GAC).

The GAC is also a percentage in per imaging area, and it is calculated at the basis of objective image as shown among table 1 using following formula: 


$$
C_{\mathrm{g}}=\frac{S_{\mathrm{g}}}{S_{\text {all }}}=\frac{\sum_{n=1}^{N_{\mathrm{sd}}} g(i, j)_{n} S_{0}}{255 N_{\text {all }} S_{0}}=\frac{\sum_{n=1}^{N_{\mathrm{sd}}} g(i, j)_{n}}{255 N_{\mathrm{all}}} \times 100 \%
$$

Where $C_{\mathrm{g}}$ is the GAC, $S_{\mathrm{g}}$ is the sediment imaging area including gray values of pixels, $n$ is an ordinal number corresponding to the $n$th number of marked points, and so $g(i, j)_{n}$ is the gray value of nth point $(i, j)$ in the objective picture, $S_{\text {all }}, N_{\text {all }}, N_{\mathrm{sd}}$ and $\mathrm{S}_{0}$ is the same as formula (2).

Definition 3 The rate between the sediment gray values squared and the total gray values squared of per image is called as gray energy density (GED) using following formula:

$$
D_{\mathrm{e}}=\frac{\sum_{n=1}^{N_{\mathrm{sd}}} g(i, j)_{n}^{2}}{\sum_{m=1}^{N_{\text {all }}} f(i, j)_{m}^{2}} \times 100 \%
$$

Where $D_{\mathrm{e}}$ is the GED, $g(i, j)_{n}$ is the gray value of $\mathrm{n}$-th point $(i, j)$ in the objective image while $f(i, j)_{m}$ is the gray value of m-th point $(i, j)$ in the original image when it is not zero, $N_{\text {all }}$ and $N_{\mathrm{sd}}$ is the same as above.

The statistical values of IAC, GAC and GED in the images from PS experiments are shown in table 1 according to above formulas. The IAC, GAC and GED are both a percentage (\%) while the related actual SSCs are both a permillage (\%). The relation curves about the statistical values of PS according to table 1 are shown in Fig. 5. Just as PS, the relation curves about the statistical values of NS are shown in Fig. 6.

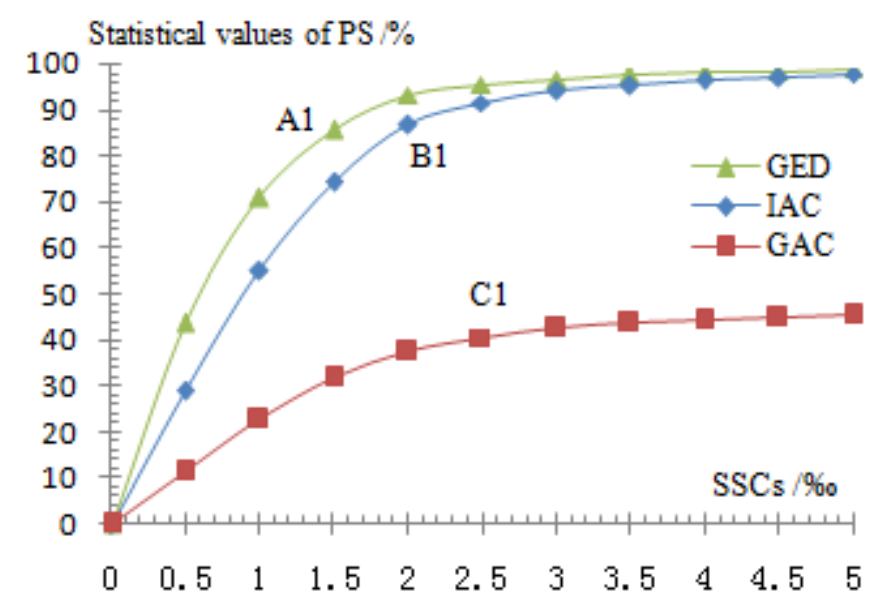

Fig. 5 Relation curves about the statistical values of PS

From Fig. 5, there is an obvious one-to-one correspondence between the GEDs and the SSCs when the concentration of PS is below $2.0 \%$. And also a clear corresponding relationship exists between the IACs and the SSCs when the concentration of PS is below $2.5 \%$, and the relationship exists between the GACs and the SSCs when the volume concentration of PS is below $4.0 \%$. The increasing tendency of the relation curves is much clearer until the point of the points of A1 (1.5 $\%, 85.5 \%)$ from the curve of GED, B1 (2.0\%, $86.8 \%)$ from the curve of IAC and C1 (2.5\%, $40.4 \%$ ) from the curve of GAC. And then the increasing tendency is slow until it is nearly no longer changed any longer when the images are full of sediments.

From Fig.6, the corresponding relationships between the statistical values and the SSCs are clear when the concentrations of NS are below $3.0 \%$ (the curve of GED), $3.5 \%$ (the curve of IAC) and $4.5 \%$ (the curve of GAC), respectively. The increasing tendency of the relation curves is quickly until the points of A2 (2.5\%, $87.7 \%)$, B2 (2.5\%, $82.2 \%)$ and $\mathrm{C} 2(3.0 \%$, $36.3 \%)$, which are from the curves of GED, IAC and GAC, respectively. Next, the increasing tendency comes to decrease until ultrasonic images are nearly filled with suspended sediments or particles. These phenomena all illustrate the relationships between B-mode ultrasound imaging signals and SSCs are steady and reliable when the volume concentration of PS and NS are low or in a certain 
concentration range.

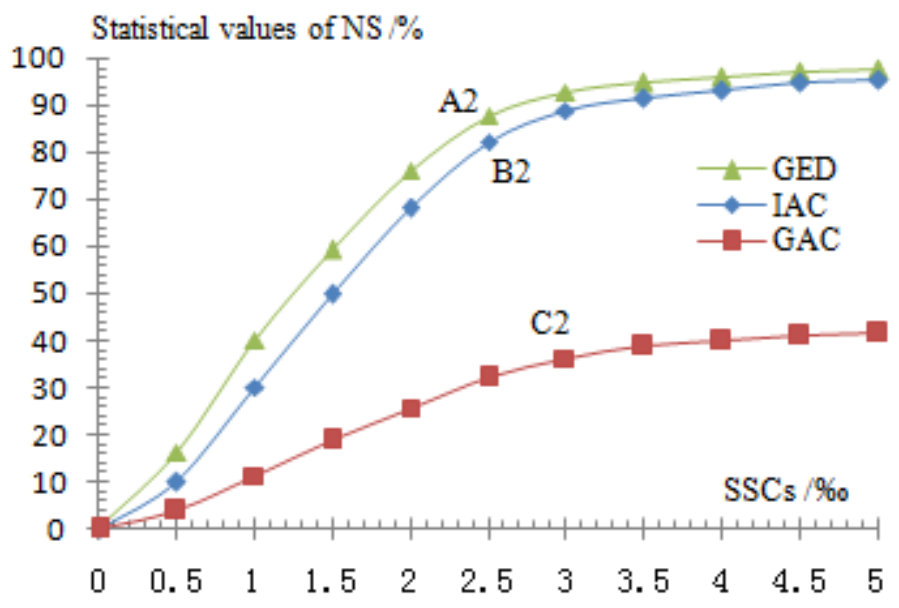

Fig. 6 Relation curves about the statistical values of NS

\subsection{Comparisons of GAC, IAC and GED}

In order to well know the inner differences of GAC, IAC and GED, their data in table 1 are normalized and shown in Fig. 7. Since the data are uniformity and comparability, Fig. 7 gives us a better understanding about the GAC, IAC and GED. In Fig. 7, the IAC of PS is divided by 97.7, the GAC of PS is divided by 45.3, and the GED of PS is divided by 98.8 while the IAC of NS is divided by 95.4 , the GAC of NS is divided by 41.6, and the GED of NS is divided by 97.6.

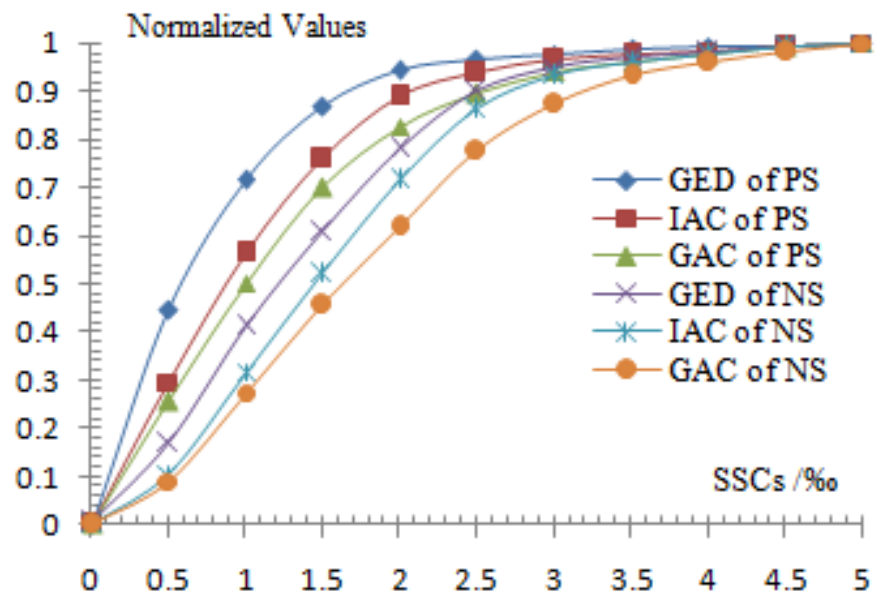

Fig. 7 The comparison of normalized relation curves

From Fig. 7, relation curves from up to down are the GED of PS, the GAC of PS, the IAC of PS, the GED of NS, the GAC of NS and the IAC of NS. It means the GED is the fastest in increasing tendency. It means the GED is the most sensitive to the change of actual SSC. The next is the GAC. The last is the IAC.

When the normalized value is up to $95 \%$ in Fig. 7, we can think it is saturated in the picture, and particles are nearly filled with B-mode images at this time. For this reason, we can see the corresponding actual volume concentrations of SSC are nearly $2.5 \%$, $4.0 \%$, $3.0 \%$ when the normalized GED, GAC, IAC of PS are up to about $95 \%$; and $3.0 \%$, $4.5 \%$, $3.5 \%$ when the normalized GED, GAC, IAC of NS are up to about $95 \%$. So the GAC is the widest in variation range compared with the IAC and the GED.

Actually, the statistical value of GAC is conveyed not only from sediment imaging area but 
also from sediment imaging gray values. Compared with A1 (1.5\%, 85.5\%), B1 (2.0\%, 86.8 \%), C1 (2.5\%, $40.4 \%)$, A2 (2.5\%, $87.7 \%)$, B2 (2.5\%, $82.2 \%)$ and C2 (3.0\%o, $36.3 \%)$, we can know, the GAC has the biggest increasing tendency compared with the IAC and the GED. Thus, the GAC is the best of all in consideration of sensitivity and anti-saturation ability. It is more sensitive to the change of actual SSC than the IAC, and has the widest variation range of all. Therefore, this paper adopts the statistical value of GAC to describe the relationship between B-mode ultrasound imaging signals and actual SSCs.

\section{Application and Discussion}

As mentioned above, the statistical value of GAC can well express the relationship between B-mode ultrasound imaging signals and actual SSCs. So, we can establish a relationship between the GAC and actual SSC, and this relationship can be used to estimate unknown SSCs and their vertical distributions in the glass flume. After a calibration test [22], the application of the relationship between the GAC and actual SSC in river engineering model was done, and also some influencing factors appeared in practical application are discussed in this part. The calibration test refers to an experimental process as the process of establishing table 1 data, which is to establish a one-to-one correspondence like Figs. 5 and 6. So, we can take the table 1 as our calibration test.

\subsection{Estimating SSC and Its Vertical Distribution}

The estimation of SSC and its vertical distribution takes these steps: Firstly, using B-mode ultrasound device to get ultrasound images of suspended sediments in sediment-laden flow, and sending the ultrasound images to a computer; Secondly, analyzing the GACs in the ultrasound images and establishing a relationship between the GAC and actual SSC by calibration test; Lastly, using the relationship to estimate unknown SSC and its vertical distribution with the help of software of Analysis SSCs (shown in Fig. 3).

Taking model plastic sediments (PS) as examples, the relationship between the GAC of PS and actual SSC is established shown as table 1 and Fig. 5. The PS's particle size is $0.32 \mathrm{~mm}$ and has been already described above. The relationship can be expressed as a fitting formula or a corresponding table. This paper takes the corresponding table as the basic data in the software, and Lagrange interpolation method is used as an addition to calculate the missing data in the table.

With the help of image processing techniques, the vertical distribution of SSCs along the change of depth is well realized by region division. According to the imaging signals and formula (3), per SSC in per estimated region can be obtained easily and quickly. An example is as shown in Fig. 8.

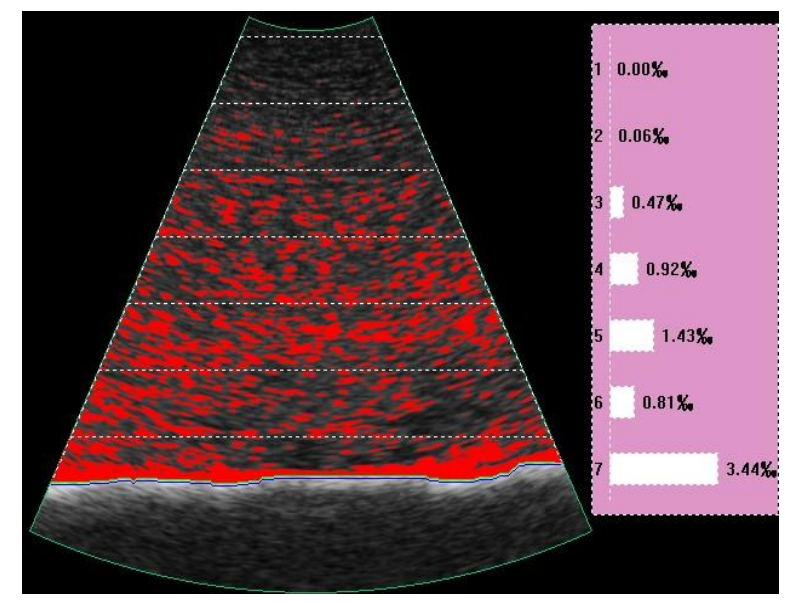

Fig. 8 The vertical distribution of SSCs along with water depth 
The estimated region of image is divided into several layers from top to bottom as needed. The GAC of each layer is calculated and turns to SSC value according to table 1. Lastly, we can get the value of SSC and its vertical distribution along with water depth, just as shown in Fig. 8. The marked pots represent suspended sediments or moving particles and the bottom of picture is the segmented topography on the bottom. The right of picture shows vertical distribution of estimated SSCs. It changes along with water depth, and shows the estimated SSC of each layer. From Fig. 8, we can see the vertical distribution of SSCs is clear and well expressed along with water depth.

To further illustrate the estimated results of SSCs and their vertical distribution, the estimated value (EV) from the result of Fig. 8 is compared with the corresponding real value (RV) from actual SSC. The RV should be measured at the same time by drying and weighing. The data of depths and SSCs are shown in table 2.

Table 2 Estimated values (EV) of vertical distribution compared with corresponding real values (RV)

\begin{tabular}{cccccccc}
\hline Depth /cm & 3.7 & 7.3 & 10.9 & 14.5 & 18.1 & 21.7 & 25.3 \\
\hline EV /\%o & 0.00 & 0.06 & 0.47 & 0.92 & 1.43 & 0.81 & 3.44 \\
RV /\%o & 0.00 & 0.05 & 0.46 & 0.93 & 1.45 & 0.79 & 3.48 \\
\hline
\end{tabular}

From table 2, the EV is approximately equal to the RV. Consequently, the application of the relationship between the GAC and the SSC described in this paper can well estimate unknown SSCs and their vertical distributions. Another, the obtained image from the B-mode ultrasound device is $768 \times 576$. When the measured depth is $25 \mathrm{~cm}$, one pixel corresponds to a distance of $0.225 \mathrm{~mm}$, so B-mode ultrasound imaging method is more suitable for a measuring depth less than $30 \mathrm{~cm}$ with a image resolution and a measuring error both less than $0.5 \mathrm{~mm}$ [4].

However, there are some problems in practical application. The relationships established by calibration test maybe have something different when test object is changed into different particle sizes or sediment materials, or when experimental condition is changed into a natural river. For example, the relation curves of PS are different from the relation curves of NS whether the GAC or the IAC and GED according to Fig. 5 and Fig. 6. Another, the focusing performance of B-mode ultrasound device is not as good as optical methods and this needs improvement. In consideration of various changed situations, the application of the relationship and the estimated data need a further study to keep it work as well under different conditions.

\subsection{Influencing Factors Analysis}

In the river model experiment, influencing factors which affect the accuracy of relationship are mainly from two aspects. On the one hand, the factors come from the test object's properties, such as particle size and sediment material. On the other hand, they come from the experimental conditions, such as ultrasonic frequency and flow velocity.

Some independent group experiments under different sediments and conditions have been carried out by using B-mode ultrasound device in this part. They are about different particle sizes, different sediment materials, different ultrasonic frequencies and different flow velocities. Some ultrasonic images are as shown in Figs. 9.

The particle size of Fig. 9 (a) is $0.12 \mathrm{~mm}$. The particle size of Fig. 9 (b) is $0.21 \mathrm{~mm}$. The sediment material of Fig. 9 (c) is pulverized coals. The sediment material of Fig. 9 (d) is wood powders. The ultrasound frequency of Fig. 9 (e) is 2 MHz. The ultrasound frequency of Fig. 9 (f) is $10 \mathrm{MHz}$. The flow velocity of Fig. 9 (g) is $3.5 \mathrm{~cm} / \mathrm{s}$. The flow velocity of Fig. 9 (h) is $12.8 \mathrm{~cm} / \mathrm{s}$. Figures 9 (a) and 9 (b) are under the same sediment material (plastic sediment), ultrasound frequency $(5 \mathrm{MHz})$ and flow velocity $(6.3 \mathrm{~cm} / \mathrm{s})$. Figures 9 (c) and 9 (d) are under the same particle size $(0.21 \mathrm{~mm})$, ultrasound frequency $(5 \mathrm{MHz})$ and flow velocity $(6.3 \mathrm{~cm} / \mathrm{s})$. Figures 9 (e) and 9 (f) are under the same particle size $(0.21 \mathrm{~mm})$, sediment material (plastic sediment) and flow 
velocity $(6.3 \mathrm{~cm} / \mathrm{s})$. Figures $9(\mathrm{~g})$ and $9(\mathrm{~h})$ are under the same particle size $(0.21 \mathrm{~mm})$, sediment material (plastic sediment) and ultrasound frequency (5 MHz). These ultrasonic images are profile maps of water flow and shown as follow:

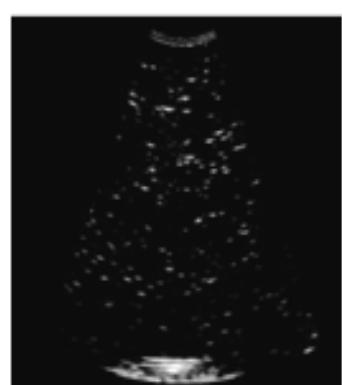

(a)

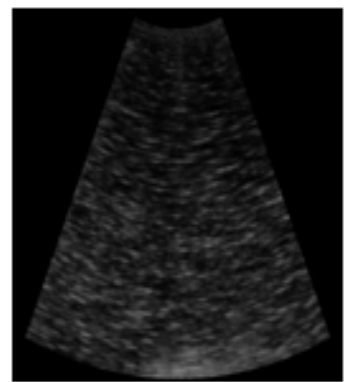

(e)

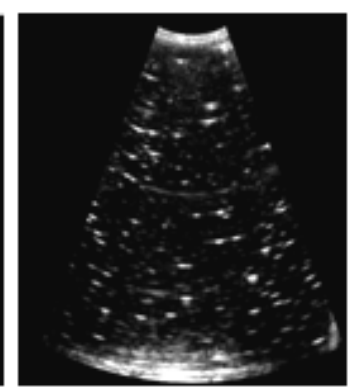

(b)

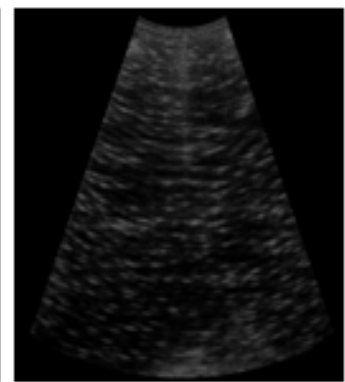

(f)

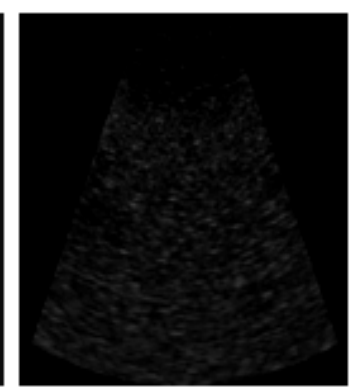

(c)

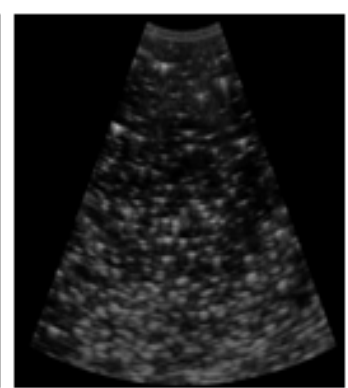

(g)

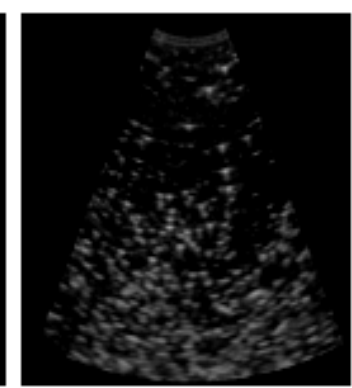

(d)

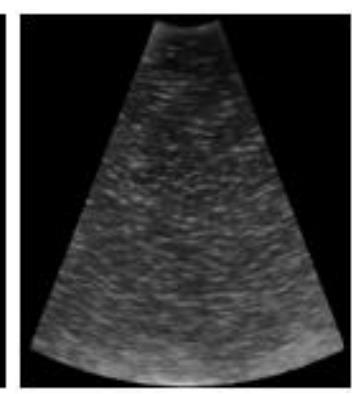

(h)

Fig. 9 Experimental images of different sediments and conditions. (a) particle size $0.12 \mathrm{~mm}$, plastic sediment, ultrasound frequency $5 \mathrm{MHz}$ and flow velocity $6.3 \mathrm{~cm} / \mathrm{s}$; (b) particle size $0.21 \mathrm{~mm}$, plastic sediment, ultrasound frequency $5 \mathrm{MHz}$ and flow velocity $6.3 \mathrm{~cm} / \mathrm{s}$; (c) sediment material pulverized coals, particle size $0.21 \mathrm{~mm}$, ultrasound frequency $5 \mathrm{MHz}$ and flow velocity $6.3 \mathrm{~cm} / \mathrm{s}$; (d) sediment material wood powders, particle size $0.21 \mathrm{~mm}$, ultrasound frequency $5 \mathrm{MHz}$ and flow velocity $6.3 \mathrm{~cm} / \mathrm{s}$; (e) ultrasound

frequency $2 \mathrm{MHz}$, particle size $0.21 \mathrm{~mm}$, plastic sediment and flow velocity $6.3 \mathrm{~cm} / \mathrm{s}$; (f) ultrasound frequency $10 \mathrm{MHz}$, particle size $0.21 \mathrm{~mm}$, plastic sediment and flow velocity $6.3 \mathrm{~cm} / \mathrm{s}$; (g) flow velocity 3.5 $\mathrm{cm} / \mathrm{s}$, particle size $0.21 \mathrm{~mm}$, plastic sediment and ultrasound frequency $5 \mathrm{MHz}$; (h) flow velocity $12.8 \mathrm{~cm} / \mathrm{s}$, particle size $0.21 \mathrm{~mm}$, plastic sediment and ultrasound frequency $5 \mathrm{MHz}$

From Figs. 9 (a) and 9 (b), if the particle size becomes bigger, the imaging spot becomes larger but the number of spots is smaller. The influence of particle size mainly affects the sediment imaging area and also gray information. From Figs. 9 (c) and 9 (d), if sediment materials are different, the imaging features of sediments also have some differences. The influence of sediment material mainly affects the corresponding curves. Different materials may match different relation curves and lead to different results. From Figs. 9 (e) and 9 (f), if the ultrasound frequency is added, the attenuation of ultrasonic becomes added and result in a weak penetration capacity. From Figs. 9 (g) and 9 (h), if the flow velocity increases, sediment imaging spots are moved with water flow and may be out of shape because high velocity leads to unsteady flow and disorder movements. The change of sediment imaging shape is a delaying phenomenon and may affect the calculation of GAC. All of these factors make a further difficulty of the application of ultrasound imaging relationship.

In order to further understand the experimental results of different sediments and conditions, the gray area concentrations (GACs) of ultrasonic images from Figs. 9 are calculated according to the definition of GAC, i.e., formula (3). Statistical values of GAC during above group experiments are shown in Figs. 10. Figure 10 (a) is the statistical results (GAC) of particle size group experiment related to Fig. 9 (a) and Fig. 9 (b). Figure 10 (b) is the statistical results of sediment material group experiment related to Fig. 9 (c) and Fig. 9 (d). In Fig. 10 (b), PS is the plastic sediment, NS is the natural sediment, PC is the pulverized coal, WP is the wood powder. Figure 10 (c) is the statistical results of ultrasound frequency group experiment related to Fig. 9 (e) and Fig. 
9 (f). Figure 10 (d) is the statistical results of flow velocity group experiment related to Fig. 9 (g) and Fig. 9 (h).

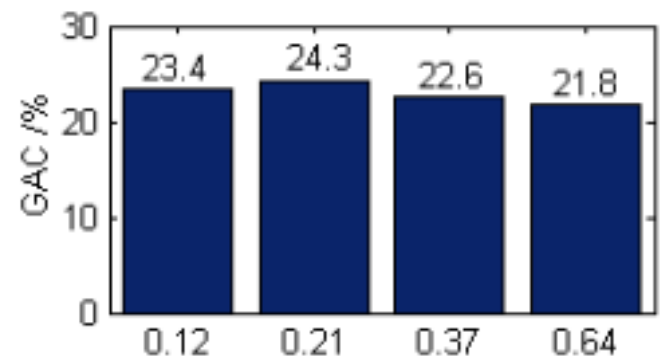

(a) Particle size $/ \mathrm{mm}$

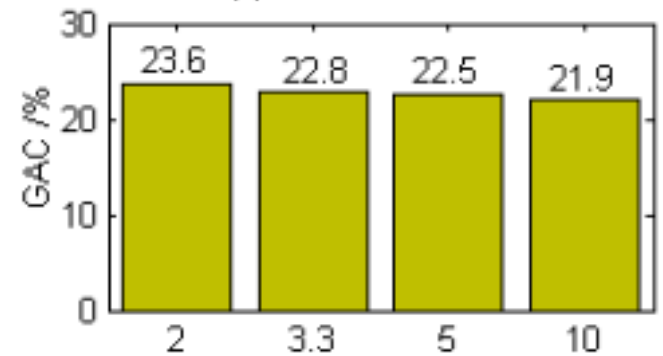

(c) Ultrasound frequency $\mathrm{MHz}$

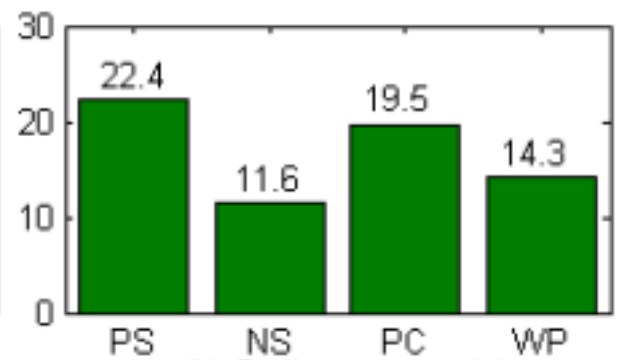

(b) Sediment material

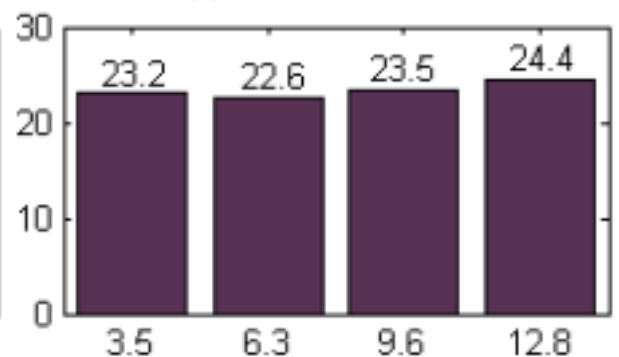

(d) Flow velocity $/ \mathrm{cm} / \mathrm{s}$

Figs. 10 The GACs of ultrasonic images under different sediments and conditions. (a) is the statistical results of particle size experiments Fig. 9 (a) and Fig.9 (b); (b) is the statistical results of sediment material experiments Fig. 9 (c) and Fig. 9 (d); (c) is the statistical results of ultrasound frequency experiments Fig. 9

(e) and Fig. 9 (f); (d) is the statistical results of flow velocity experiments Fig. 9 (g) and Fig. 9 (h)

From Figs. 10, the statistical results of GAC from above group experiments have shown some differences under the same sediments or conditions. Figures 10 (a) and 10 (b) show that different particle sizes or different sediment materials can result in different GACs. Figures 10 (c) and 10 (d) show that the addition of GAC can be along with the increase of flow velocity or the decrease of ultrasound frequency within a certain range. However, these factors are not independent each other during the application of GAC. They always exist and affect each other that can't clear explained at present experimental condition [23, 24].

In fact, the influences of particle size, sediment material, ultrasound frequency and flow velocity on the GAC's statistical results are common and also exist by using other acoustical methods $[1,25,26]$. They contact and contain each other, and are difficult to sort out their internal connections. In order to simplify this cumbersome process, we usually adopt a common method in sediment concentration measurement field. That is the specialized object with the specialized calibration test. The calibration test is an experimental process as the process of establishing table 1 data. Consequently, it is necessary to go on a specialized calibration test before the application of GAC relationship, especially when experimental object is changed. In other words, establishing a corresponding table between B-mode ultrasound imaging signals and actual SSCs is necessary before the application of these relationships. In this way, we can get a more precise and reliable result when using B-mode ultrasound imaging method to measure SSC and its vertical distribution in river model test.

\section{Conclusion}

In this paper, we proposed some corresponding relationships between the B-mode ultrasound imaging signals and the actual SSCs, and the practical application of GAC to estimate low SSC and vertical distribution. Our conclusions are listed as follow: 
(1) Three kinds of corresponding relationships: gray area concentration (GAC), imaging area concentration (IAC) and gray energy density (GED), but the GAC is better than the IAC and the GED because of sensitivity and variation range.

(2) These relationships can be affected by different particle sizes, sediment materials, ultrasound frequencies and flow velocities. When we use these relationships to estimate unknown SSCs, a corresponding table should be established first between B-mode ultrasound imaging signals and actual SSCs by specialized calibration test.

(3) The application of corresponding relationships, such as the GAC, can well realize the estimation of unknown SSC and its vertical distribution in the glass flume of river model tests. This method can realize the real-time monitor and analysis of SSC in sediment-laden flow

(4) B-mode ultrasound imaging method for the estimation of SSC has the advantages of direct observation, high sensitivity and good real-time. This is a new way to realize the dynamic analysis of suspended sediment transport process.

\section{References:}

[1] Jaafar W, Fischer S, Bekkour K. Velocity and turbulence measurements by ultrasound pulse Doppler velocimetry[J]. Measurement, 2009, 42(2): 175-182.

[2] Crapper M, Bruce T, Gouble C. Flow field visualization of sediment-laden flow using ultrasonic imaging[J]. Dynamics of Atmospheres and Oceans, 2000, 31(1): 233-245.

[3] Gungor M A, Karagoz I. The homogeneity map method for speckle reduction in diagnostic ultrasound images[J]. Measurement, 2015, 68: 100-110.

[4] Zou X, Ma Z, Hu W, et al. B-mode ultrasound imaging measurement and 3D reconstruction of submerged topography in sediment-laden flow[J]. Measurement, 2015, 72: 20-31..

[5] Pedocchi F, García M H. Acoustic measurement of suspended sediment concentration profiles in an oscillatory boundary layer[J]. Continental Shelf Research, 2012, 46: 87-95..

[6] Osborne P D, Vincent C E, Greenwood B. Measurement of suspended sand concentrations in the nearshore: field intercomparison of optical and acoustic backscatter sensors[J]. Continental Shelf Research, 1994, 14(2): 159-174..

[7] Hamilton L J, Shi Z, Zhang S Y. Acoustic Backscatter Measurements of Estuarine Suspended Cohesive Sediment Concentration Profiles [J]. Journal of Coastal Research. 1998, 14(4): 1213-1224.

[8] Holdaway G P, Thorne P D, Flatt D, et al. Comparison between ADCP and transmissometer measurements of suspended sediment concentration[J]. Continental shelf research, 1999, 19(3): 421-441..

[9] Betteridge K F E, Williams J J, Thorne P D, et al. Acoustic instrumentation for measuring near-bed sediment processes and hydrodynamics [J]. Journal of Experimental Marine Biology and Ecology. 2003, 285-286(0): 105-118.

[10] Thorne P D, Hanes D M. A review of acoustic measurement of small-scale sediment processes [J]. Continental Shelf Research. 2002, 22(4): 603-632.

[11] Stolojanu V, Prakash A. Characterization of slurry systems by ultrasonic techniques [J]. Chemical Engineering Journal. 2001, 84(3): 215-222.

[12] Latour S, Gallot T, Catheline $S$, et al. Ultrafast ultrasonic imaging of dynamic sliding friction in soft solids: The slow slip and the super-shear regimes[J]. EPL (Europhysics Letters), 2011, 96(5): 59003.

[13] Ma Z, Zou X, Zhao X, et al. The Measurement of Low Sediment Concentration Based on B-Mode Ultrasound Images [J]. Journal of Basic Science and Engineering. 2013, 21(4): 796-803.

[14] Ma Z, Zou X, Zhao X, et al. A method of measuring sediment incipient velocity based on B-scan 
ultrasound imaging technique [J]. Advance in Water Science. 2015, 26(01): 59-65.

[15] Song H, Wang C, Zou X, et al. An Extracting Method of Underwater Model Topography Based on B-mode Ultrasound Images [J]. Journal of Basic Science and Engineering. 2015(02): 225-232.

[16] Bamberger J A, Greenwood M S. Using ultrasonic attenuation to monitor slurry mixing in real time[J]. Ultrasonics, 2004, 42(1): 145-148.

[17] Skwarek V, Windorfer H, Hans V. Measuring pulsating flow with ultrasound[J]. Measurement, 2001, 29(3): 225-236

[18] Dadashnialehi A, Moshiri B. Online monitoring of transit-time ultrasonic flowmeters based on fusion of optical observation[J]. Measurement, 2011, 44(6): 1028-1037.

[19] Chen J, Cheng C, Yeh C. Skin-scanning technique for superficial blood flow imaging using a high-frequency ultrasound system [J]. Ultrasonics. 2014, 54(1): 241-246.

[20] Zhou B, Fraser K H, Poelma C, et al. Ultrasound Imaging Velocimetry: Effect of Beam Sweeping on Velocity Estimation [J]. Ultrasound in Medicine \& Biology. 2013, 39(9): 1672-1681.

[21] Blachowski B, Swiercz A, Gutkiewicz P, et al. Structural damage detectability using modal and ultrasonic approaches[J]. Measurement, 2016, 85: 210-221.

[22] Shukla A, Prakash A, Rohani S. Particles settling studies using ultrasonic techniques [J]. Powder Technology. 2007, 177(2): 102-111.

[23] Marinozzi F, Branca F P, Bini F, et al. Calibration procedure for performance evaluation of clinical Pulsed Doppler Systems[J]. Measurement, 2012, 45(5): 1334-1342.

[24] Alvarenga A V, Teixeira C A D, von Krüger M A, et al. Uncertainty evaluation from non-invasive estimation of temperature variation using B-mode ultrasonic images from a plastic phantom[J]. Measurement, 2015, 69: 189-194..

[25] Minella J P G, Merten G H, Reichert J M, et al. Estimating suspended sediment concentrations from turbidity measurements and the calibration problem[J]. Hydrological processes, 2008, 22(12): 1819-1830..

[26] Wren D G, Langendoen E J, Kuhnle R A. A note on acoustic measurements of turbulence, suspended sediment, and bed forms in mobile-bed experiments [J]. Journal of Hydro-environment Research. 2013, 7(2): 1-10. 

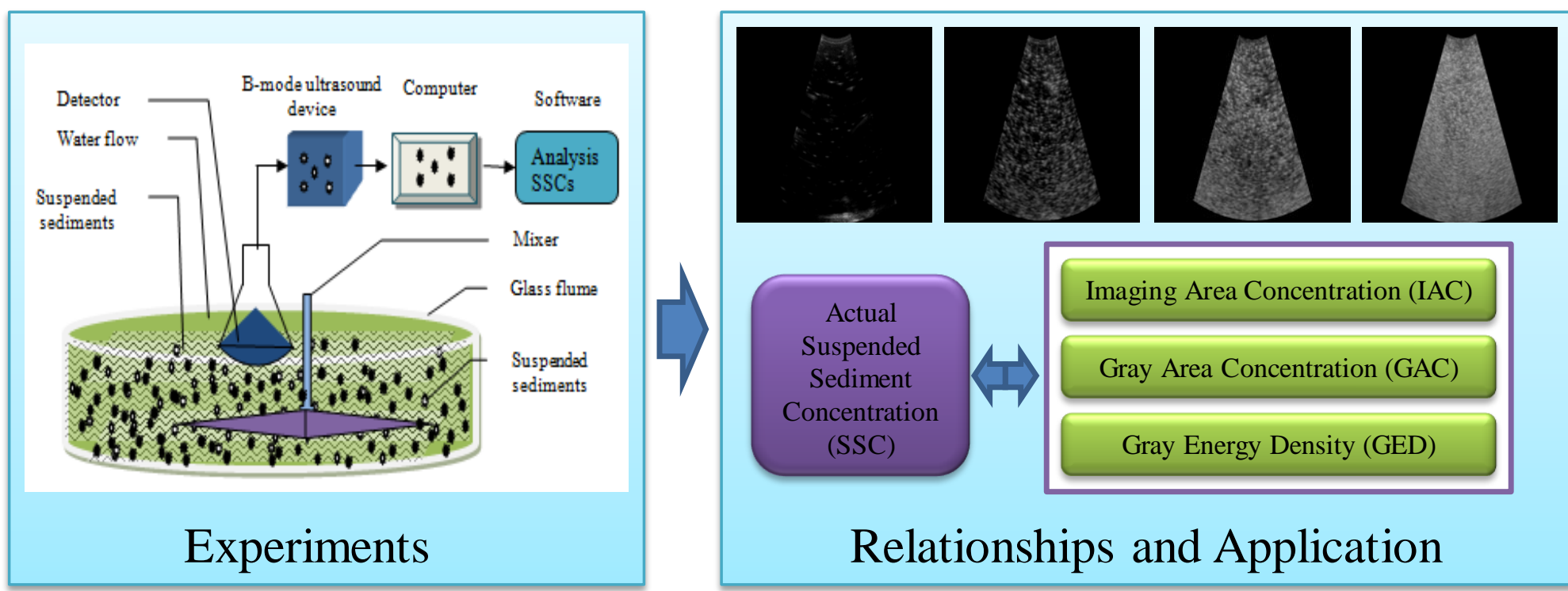

Imaging Area Concentration (IAC)

Gray Area Concentration (GAC)

Gray Energy Density (GED)

Relationships and Application 